\title{
Supercontinuum Generation in Hydrogenated Amorphous Silicon Waveguides in the Femtosecond Regime
}

\author{
S.-P. Gorza ${ }^{1}$, F. Leo ${ }^{2}$, B. Kuyken², S. K. Selvaraja ${ }^{2}$, G. Roelkens ${ }^{2}$, R. Baets ${ }^{2}$, \\ Ph. Emplit ${ }^{1}$, S. Massar ${ }^{3}$ and J. Safioui ${ }^{1}$ \\ ${ }^{1}$ Service OPERA, Université Libre de Bruxelles (ULB), 50 Avenue F. D. Roosevelt, CP 194/5, B-1050 Bruxelles, \\ Belgium \\ ${ }^{2}$ Photonics Research Group, INTEC Department, Ghent University-IMEC, Sint-Pietersnieuwstraat 41, 9000 Ghent, \\ Belgium \\ ${ }^{3}$ Laboratoire d'Information Quantique, Université Libre de Bruxelles (ULB), 50 Avenue F. D. Roosevelt, CP 225, \\ B-1050 Bruxelles, Belgium \\ sgorza@ulb.ac.be
}

\begin{abstract}
Supercontinuum generation in CMOS compatible hydrogenated amorphous silicon waveguides with femtosecond pulses at telecommunication wavelengths is experimentally studied. It is shown that stable $540 \mathrm{~nm}$ broad supercontinua can be obtained in $1 \mathrm{~cm}$-long waveguides.
\end{abstract}

(C) 2014 Optical Society of America

OCIS codes: (130.4310) Nonlinear; (320.6629) Supercontinuum generation.

Extreme spectral broadening that occurs when narrowband input pulses propagate in nonlinear optical media is known as supercontinuum (SC) generation [1]. In nonlinear bulk media, ultrahigh pulse energy delivered by cumbersome sources was required to observe this spectral widening. However, with the advent of guiding structures such as optical fibers, SC generation is possible with much lower pulse energy (and even with continuous waves) thanks to dispersion engineering and effective nonlinearity enhancement. Nevertheless, the integration of such sources still remains an important topic in photonic research owing to their possible applications. Hydrogenated amorphous silicon (a-Si:H) waveguides, thanks to their CMOS compatibility and high nonlinearity, are one of the most promising ultra-compact nonlinear devices for SC generation.

In this work we focus on the generation of SC with femtosecond pulses in a-Si:H waveguides at telecommunication wavelengths around $1.55 \mu \mathrm{m}$. The advantage of this material over crystalline silicon is its larger bandgap of about $1.6 \mathrm{eV}$. This larger value results in lower two-photon absorption (TPA) and TPA induced free-carrier absorption, which are known to strongly limit the efficiency of nonlinear interactions [2]. SC generation has been recently observed in a-Si:H waveguide with picosecond pulses [3]. However in this latter work material degradation has been reported and we expect this degradation to be reduced in the femtosecond regime.

Experiments were performed in $1 \mathrm{~cm}$-long waveguides (cross-section $220 \times 500 \mathrm{~nm}^{2}$ ) fabricated using deep UV optical lithography and dry etching [4]. At telecommunication wavelengths, these waveguides are characterized by a linear loss of $\alpha=3 \mathrm{~dB} / \mathrm{cm}$ and a nonlinear parameter $\gamma=(740+\mathrm{i} 31) \mathrm{W}^{-1} \mathrm{~m}^{-1}$. At $1550 \mathrm{~nm}$, the dispersion is anomalous and the GVD value has been estimated to be $\beta_{2}=-2 \mathrm{ps}^{2} / \mathrm{m}$ [5]. The source is an OPO laser delivering pulses of about $150 \mathrm{fs}$ around $1550 \mathrm{~nm}$ with a repetition rate of $82 \mathrm{MHz}$. The laser beam was coupled into the waveguide by means of a $\times 60$ microscope objective to excite the quasi-TE mode of the waveguide. At the waveguide output, the light was collected with a lensed fiber $(\mathrm{NA}=0.4)$.

Typical SC spectra at the waveguide output are reported in Fig.1(a). At an on chip peak power of $18 \mathrm{~W}$, i.e. a pulse energy of $2.7 \mathrm{pJ}$, the spectrum extends from $1380 \mathrm{~nm}$ to $1920 \mathrm{~nm}$ at $-20 \mathrm{~dB}$ from the peak maximum [see red curve in Fig.1(a)]. This spectrum is likely the result of self-phase modulation, soliton fission and dispersive wave generation. This latter phenomenon is an energy transfer from a soliton to a narrow frequency band in the normal GVD regime, and the peak around $1840 \mathrm{~nm}$ at $2 \mathrm{~W}$ input power is probably the signature of such a dispersive wave. The detailed physical mechanisms underlying the $\mathrm{SC}$ in a-Si:H nanowires are currently being investigated through numerical simulations of the pulse propagation.

For large input pump power, a-Si:H waveguides suffer from optical degradation resulting in a decrease of the strength of nonlinear effects over time. This decrease is due to an increase of both linear and nonlinear losses. This 

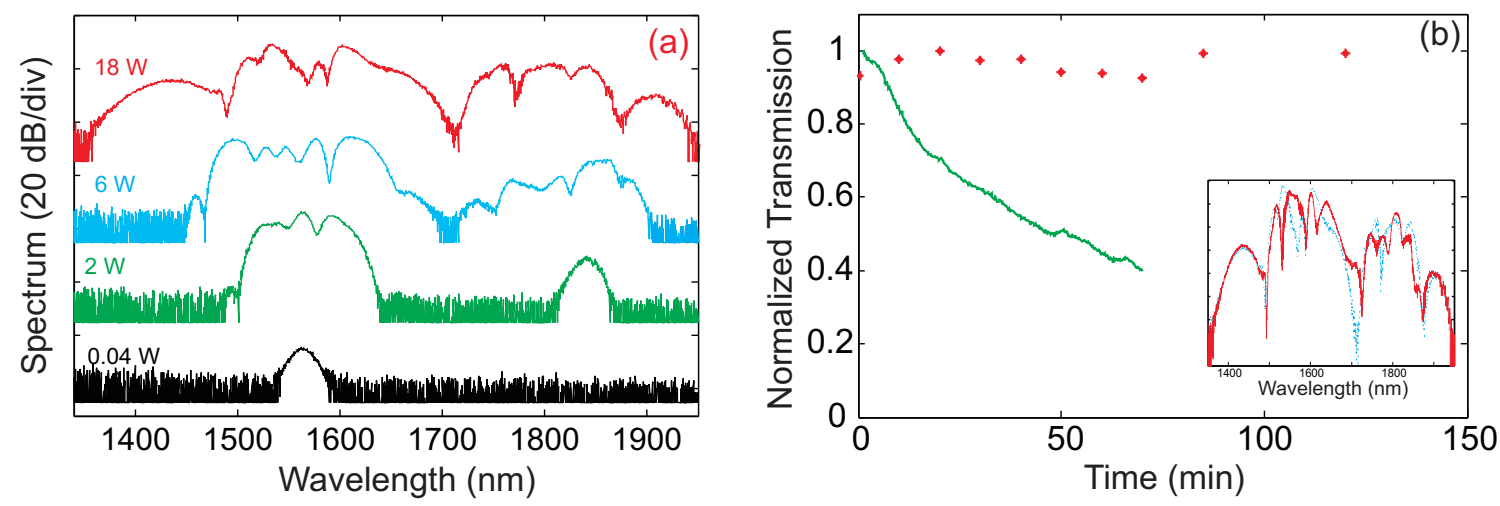

Fig. 1: (a) Experimental spectra recorded at the output of a $1 \mathrm{~cm}$-long a-Si:H nanowire, for increasing on chip peak power. (b) Time evolution of the transmission, normalized to its largest value. Transmission recorded in the same conditions as for the top curve in panel (a) (red cross), and with 4 ps pulses of $3 \mathrm{~W}$ on chip peak power (green curve). The two measurements were performed in identical waveguides. The inset shows the SC spectra at $t=0$ (dashed blue) and after $120 \mathrm{~min}$ (solid red) in the femtosecond regime. The small differences between these two spectra are due to laser fluctuations.

degradation can clearly be seen in the time evolution of the transmission coefficient of picosecond pulses in Fig.1 (b). In this ps regime, the degradation also reduces the SC bandwidth over time [3]. However, no degradation was observed with femtosecond pulses even for larger average power and pulse energy than for the experiment reported in [3]. The transmission, recorded during two hours, shows no measurable decrease of its value. We can thus reasonably expect that both the linear and nonlinear absorptions remained unchanged during this time interval. The material stability is remarkably confirmed in the spectra recorded at two hours interval [see inset in Fig.1 (b)].

Coherence (i.e. pulse-to-pulse variations) in the generated SC is related to the sensitivity of the broadening mechanism to the precise input pulse condition. For short pulses around $100 \mathrm{fs}$, it is known that the degree of coherence can be interpreted as the result of a competition between soliton fission and modulation instability [1]. In this regime, the coherence of the SC at the output of the waveguide is thus strongly related to the pulse duration (and its bandwidth), the dispersion properties of the waveguide at the pump wavelength and the waveguide length. Preliminary measurements in the $1500 \mathrm{~nm}-1650 \mathrm{~nm}$ range show that the SC reported in Fig. 1(a) is coherent, which is encouraging for applications of SC generated on chip at telecommunication wavelengths.

In conclusion, we have experimentally demonstrated the generation of a supercontinuum at telecommunication wavelengths in a-Si:H waveguides using 150 fs pulses. The SC remains stable over hours contrary to the SC observed in identical waveguides with picosecond pulses. Numerical simulations of the SC generation are currently undertaken to confirm our interpretation of the SC generation in a-Si:H waveguides.

\section{References}

1. J. M. Dudley, G. Genty and S. Coen, "Supercontinuum generation in photonic crystal fiber," Rev. Mod. Phys 78, 1135-1184 (2006).

2. J. Matres, G. C. Ballesteros, P. Gautier, J-M Fdli, J. Mart, and C. J. Oton, "High nonlinear figure-of-merit amorphous silicon waveguides," Opt. Express 21, 3932-3940 (2013).

3. J. Safioui, F. Leo, B. Kuyken, S.-P. Gorza, S. K. Selvaraja, R. Baets, Ph. Emplit, G. Roelkens, and S. Massar, "Supercontinuum generation in hydrogenated amorphous silicon waveguides at telecommunication wavelengths," Optics Express, to be published.

4. S. K. Selvaraj, E. Sleeckx, M. Schaekers, W. Bogaerts, D. V. Thourhout, P. Dumon, R. Baets, "Low-loss amorphous silicon-on-insulator technology for photonic integrated circuitry," Optics Communications 282, 17671770 (2009).

5. B. Kuyken, S. Clemmen, S. K. Selvaraja, W. Bogaerts, D. V. Thourhout, Ph. Emplit, S. Massar, G. Roelkens, and R. Baets, "On-chip parametric amplification with $26.5 \mathrm{~dB}$ gain at telecommunication wavelengths using CMOS-compatible hydrogenated amorphous silicon waveguides," Optics letters 36, 552-554 (2011). 